\title{
Errors in oblique cephalometric radiographic projections of the edentulous mandible. Part I: Geometric errors
}

\author{
W. H. A. Steen, Drs.* \\ University of Utrecht, School of Dentistry, Utrecht, The Netherlands
}

$S_{\text {tute }}$ tudies to evaluate the effect of dental treatment on resorption of the edentulous mandible are generally carried out with radiographic techniques. Wical and Swoope ${ }^{1}$ and Van Waas ${ }^{2}$ used orthopantographs. They found that it is extremely difficult to obtain reproducible images in a longitudinal study. Van Aken $^{3,4}$ and Lund and Manson-Hing ${ }^{5}$ found that lateral cephalometric projection is more reliable. Tallgren, ${ }^{6,7}$ Tallgren et al., ${ }^{8}$ Carlsson and Persson, ${ }^{9}$ Atwood and Coy, ${ }^{10}$ and others have also applied this technique. One restriction is that the images permit only exact measurements of the anterior region of the mandible because of the problem of overprojection of the left and right sides of the mandible. Cartwright and Harvold ${ }^{11}$ introduced the oblique projection as a means of diminishing overprojection.

With the oblique cephalometric technique the midsagittal plane of the patient's head makes a certain angle $\alpha$ to the axis of the radiographic rays. The radiographic film remains perpendicular to the axis. By varying the angle $\alpha$ one can obtain clear views of different parts of the mandible without overprojection of the opposite side of the mandible or the cervical vertebrae.

Barber et al. ${ }^{12}$ evaluated the oblique cephalometric technique for its usefulness in orthodontic studies. They took a random selection of 10 skulls that varied in maturity from infancy to adulthood and compared horizontal and vertical dimensions of the upper and lower jaw on radiograms at a 45-degree oblique projection. The tubehead was fixed in relation to the cephalostat at a distance of $1500 \mathrm{~mm}$ from target to the midsagittal plane. The mean enlargement percentages for vertical measurements of the mandible ranged between $+0.64 \%$ and $+5.15 \%$ depending on the region measured. The corresponding horizontal values ranged

\footnotetext{
* Research Associate, Department of Prosthodontics.

tThe midsagittal plane was defined as the plane perpendicular to the line connecting the two ear fixation pins in the left and right external auditory meatus and at equal distances from the pins. ${ }^{3}$
}

from $-3.2 \%$ to $+4.17 \%$. The authors only listed averages without giving information about the percentages for each separate skull. They concluded that the enlargement in the oblique projection was not greater than that encountered in the more universally employed lateral cephalometric projection.

Winter et al. ${ }^{13}$ made measurements on oblique cephalometric radiograms with the patient's edentulous mandible in the rest position with angles of 35 degrees and 325 degrees for the left and right side projection, respectively. Data on the resorption measured from the radiograms were derived from zones $30 \mathrm{~mm}$ posterior to the lateral incisor. However, the authors did not publish data on the reproducibility of these measurements.

This radiographic technique made it obvious that it is important to orient the head in the cephalostat with the porion-subnasal plane in a horizontal position. The lower mandibular border should have an inclination of approximately 15 degrees to avoid overprojection of other structures of the skull. To fulfill these requirements a Wehmer cephalostat (B.F. Wehmer, Inc., Franklin Park, Ill.) was modified by the author. ${ }^{16}$ With this technique when the angle $\alpha$ is approximately 30 degrees as indicated in Fig. 1, $A$, neither the cervical vertebrae nor the right side of the mandible is projected over the left frontal region. Fig. $1, B$, shows a clear view of the lateral part when angle $\alpha$ is 45 degrees.

The purpose of this study (Part I) was to evaluate the geometric errors of the method to decide on the applicability of the technique in a longitudinal study. The aim of this clinical study is to develop a measuring technique to compare the patterns of resorption in the mandible for different types of prosthodontic treatment. In Part II the technical and exposure data will be given, and the total error that may arise is calculated by measuring sections of the radiographs.

\section{GEOMETRIC ERRORS}

Because of practical limitations with respect to exposure times and the available space in the average 


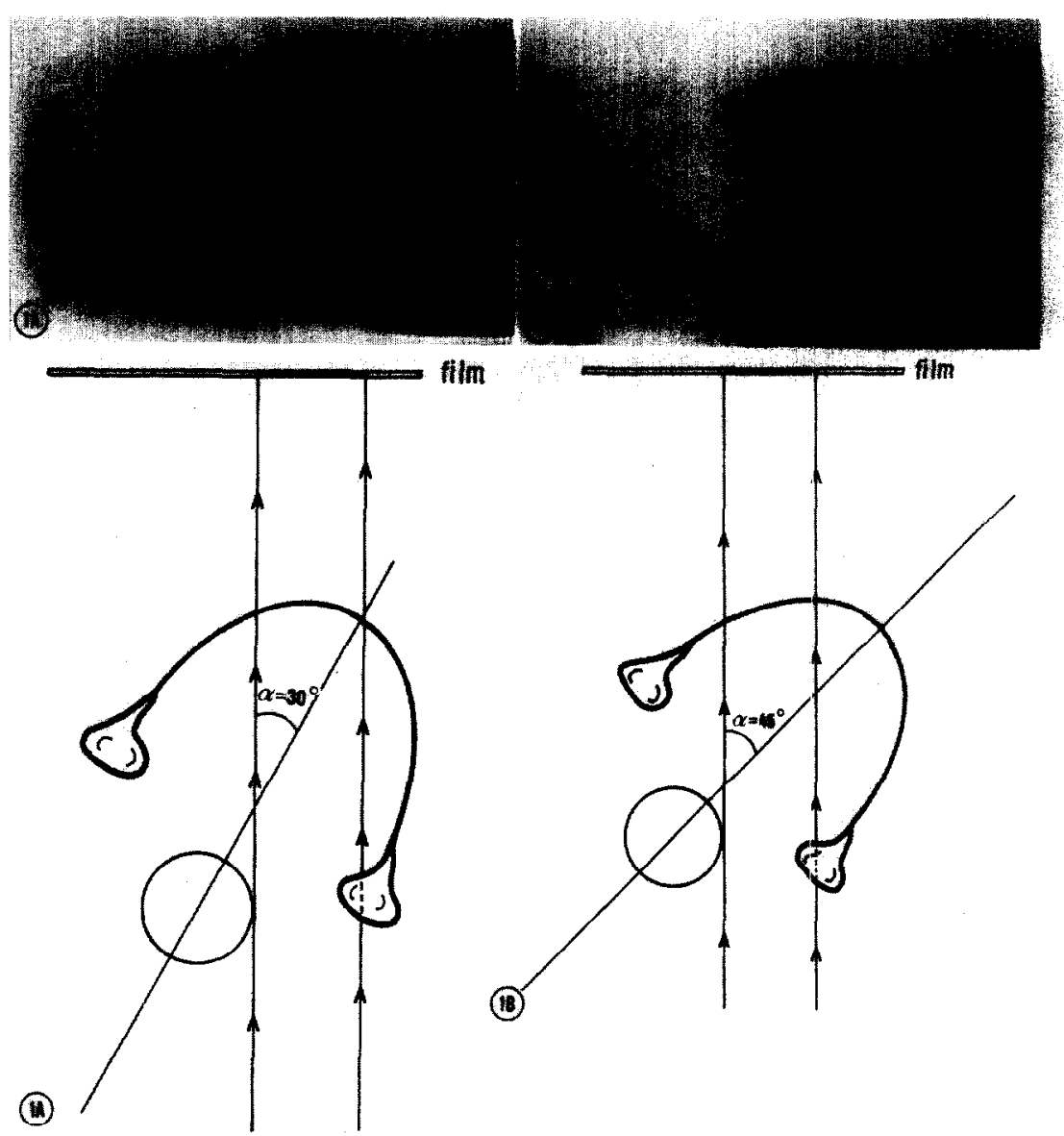

Fig. 1. Top, Radiographs. Bottom, Schematic drawings. A, When angle $\alpha$ between $x$-ray beam and midsagittal plane of patient approximates 30 degrees as indicated, neither cervical vertebrae nor right side of mandible is projected over left frontal region. $B$, Clear view of lateral section when angle $\alpha$ is 45 degrees.

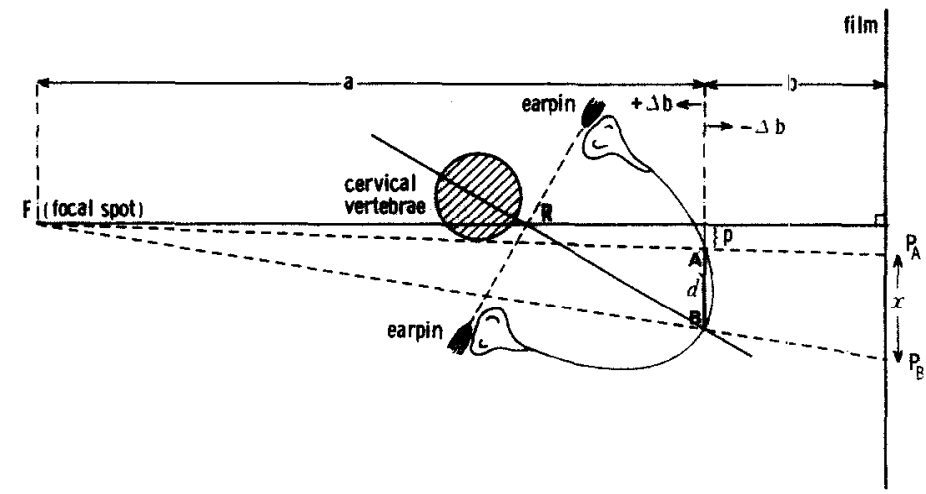

Fig. 2. Schematic drawing of mandible and projecting radiographic rays. $\chi=$ Image on radiographic film of part $A B(=d)$ of mandible.

radiology room, the distance from the focal spot to the object ranges in most instances between $1500 \mathrm{~mm}$ and $6000 \mathrm{~mm}$. As a consequence, when the object is placed parallel to the film, the projecting rays will diverge, which results in an enlarged image. In our calculations we will consider that in the 30 -degree oblique projection the radiographic film is parallel with the frontal region of the mandible, and in the 45-degree oblique projection it is parallel with the lateral region.

In Fig. 2 a designated part of the mandible $A B$ has a 


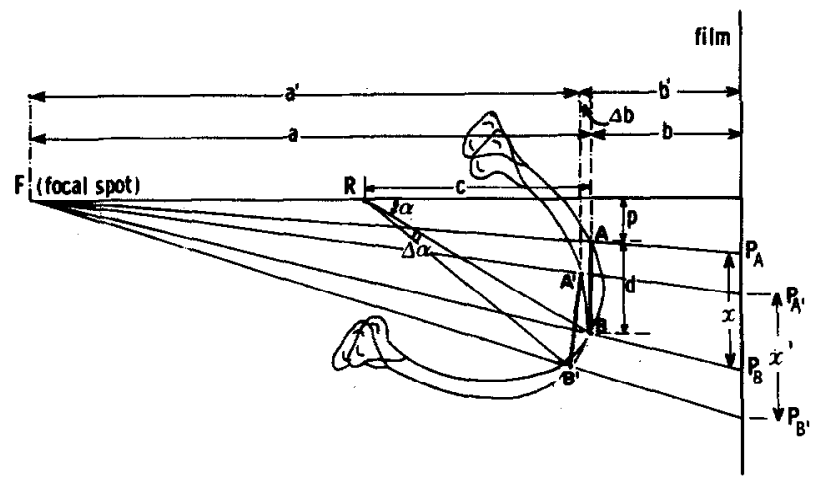

Fig. 3. Schematic drawing of a horizontal section of mandible illustrating factors producing horizontal geometric errors. Image of part $A B$ is indicated by $\chi . A B$ becomes $A^{\prime} B^{\prime}$. when patient's head is rotated from desired position through an angle $\Delta \alpha . \chi^{\prime}$ is image of $A^{\prime} B^{\prime}$ on radiographic film. Difference between $\chi$ and $\chi^{\prime}$ is error produced by angle $\Delta \alpha$.

length $\mathrm{d}$ and is located at the distance $\mathrm{p}$ from the central axis of the radiographic beam. The projection of $A B$ on the radiogram is indicated by $P_{A} P_{B}(x)$. The length of $\chi$ is determined with formula No. 1 .

$$
\frac{a+b}{a} d
$$

In this formula $\mathrm{a}$ and $\mathrm{b}$ are, respectively, the distances from the focal spot to the object and from the object to the radiogram. The difference between $\mathrm{d}$ and $\chi$ is the increase in length as registered on the radiograph. This increase is independent of the distance $p$ and will be larger when $b$ is larger and/or $a$ is smaller. When $F$ is at an infinite distance from the patient, then all projecting rays are parallel. In this theoretical situation there will be no enlargement.

In this investigation the factors influencing the length $\chi$ for a fixed arrangement of focal spot, cephalostat, and radiographic film in oblique cephalometric projections are studied. Blurring from a focal spot of $1.5 \mathrm{~mm}$ is less than $0.1 \mathrm{~mm}\left(\frac{1.5 \mathrm{~b}}{\mathrm{a}}\right)$. Therefore, simplification of the problem is justified by assuming that the focal spot has no dimension. Variations in the length of $\chi$ are produced by (1) differences in the distance between object and radiographic film, (2) horizontal rotation of the mandible (around a vertical axis), and (3) vertical rotation of the mandible (around a horizontal axis).

\section{Differences in the distance between object and radiographic film}

In a fixed arrangement of the radiograph machine, cephalostat, and film, the sum of $a$ and $b$ has a constant value; but because of individual differences in anatomy,
Table I. Enlargement percent $\left(\frac{x-d}{d} 100\right)$

of $d$ for different values of $a$ and $b$ (in millimeters)

\begin{tabular}{ccccccccc}
\hline & \multicolumn{3}{c}{ Frontal region } & & \multicolumn{3}{c}{ Lateral region } \\
\cline { 2 - 3 } \cline { 6 - 7 } & $\mathbf{b}=\mathbf{6 0}$ & $\mathbf{b}=\mathbf{7 5}$ & $\mathbf{b}=\mathbf{9 0}$ & $\mathbf{b}=\mathbf{7 0}$ & $\mathbf{b}=\mathbf{8 5}$ & $\mathbf{b}=\mathbf{1 0 0}$ \\
\hline $\mathrm{a}+\mathrm{b}=1575$ & 4.0 & 5.0 & 6.1 & & 4.7 & 5.7 & 6.8 \\
$\mathrm{a}+\mathrm{b}=3075$ & 2.0 & 2.5 & 3.0 & & 2.3 & 2.8 & 3.4 \\
$\mathrm{a}+\mathrm{b}=6075$ & 1.0 & 1.25 & 1.5 & & 1.2 & 1.4 & 1.7 \\
\hline
\end{tabular}

the separate values of $a$ and $b$ vary slightly for each subject, which result in differences in the enlargement factor (Fig. 2). Moreover, because of the shape of the mandible, the values for $b$ are different for the 30degree and 45-degree oblique projection in the same subject.

To obtain more information, the variations of $b$ were measured in a sample of six adult subjects who were selected because of the differences in their facial structures. With a distance of $150 \mathrm{~mm}$ between the center of the cephalostat ( $R$ ) and the radiographic film, in the 30-degree oblique projection, the values for $b$ ranged from 60 to $90 \mathrm{~mm}$. In the 45-degree oblique projection, the figures were approximately $10 \mathrm{~mm}$ more. This implies an average value for distance $b$ of $75 \mathrm{~mm}$ in the 30 -degree and $85 \mathrm{~mm}$ in the 45-degree oblique projection.

When the relative measurements are compared on subsequent radiograms of one patient, and the same oblique projection is used, there is no need to calculate the enlargement because it has a constant value. When oblique radiograms of different patients are compared, the enlargement factor differs because of differences in 


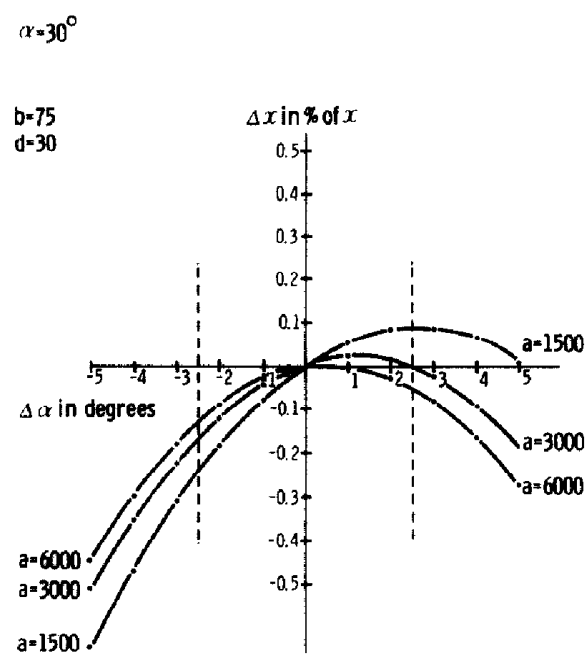

(4)

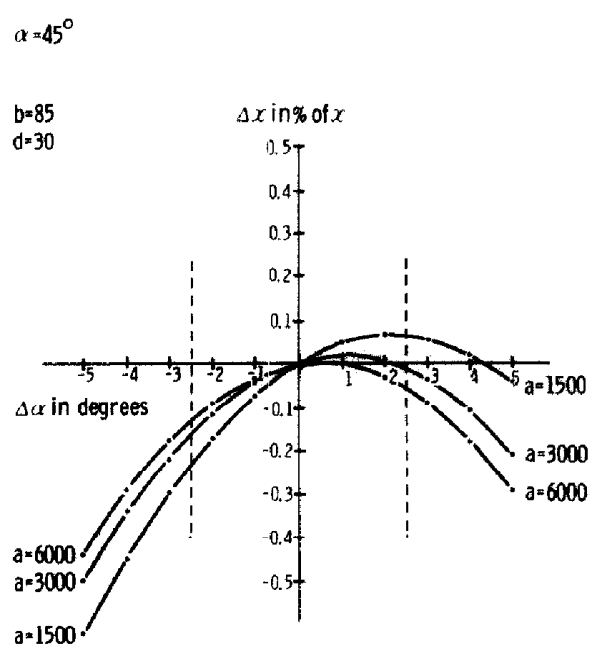

(5)

Figs. 4 and 5. Geometric error $\Delta \chi$ on oblique cephalometric films has been expressed as percentage of $\chi$ due to positive and negative horizontal rotations of mandible.

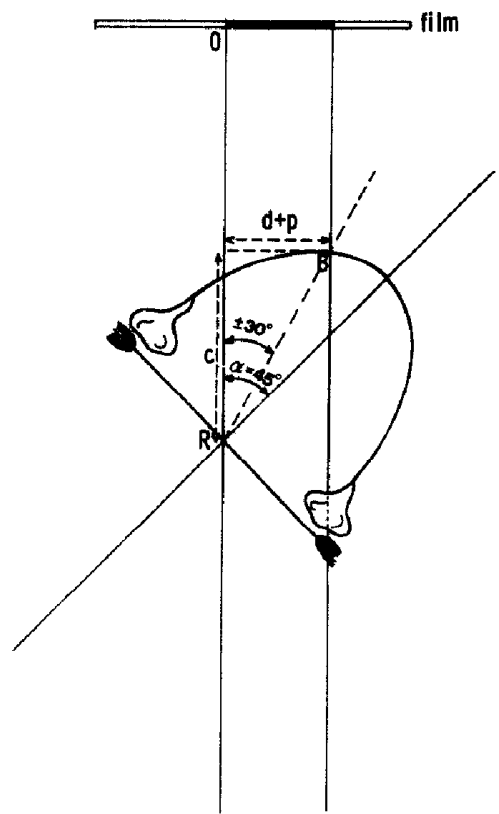

Fig. 6. Mean value of $(d+p)$ in 45-degree situation (see text).

the values of $a$ and $b$. In Table I, enlargement percentages $\left(\frac{x-d}{d} 100\right)$ of $d$ are tabulated for different values of $a$ and $b$ with formula No. 1 .

\section{Horizontal rotation of the mandible (ad 2)}

When the patient is placed in a cephalostat, the head may rotate from the desired position immediately before or at the moment of exposure. This is due to the flexibility of the soft tissues between the fixation pins of the cephalostat" and the bony structures of the skull, and to the play in the brackets of the cephalostat (Fig. 3).

In Fig. $3 \mathrm{AB}$ (d) represents a horizontal part of the mandible; $P_{A}$ and $P_{B}$ are, respectively, the projections on the radiographic film of $A$ and $B$. The image $P_{A} P_{B}$ is indicated by $\chi$. The angle between the midsagittal plane of the patient's head and the central axis of the radiographic beam is indicated by $\alpha$. The vertical axis of rotation is R. For convenience it is accepted that this point is situated at an equal distance from both ear fixation pins. The deviation of the angle $\alpha$ around rotating center $\mathrm{R}$ is called $\Delta \alpha$. After $\mathrm{AB}$ has rotated, the new position is represented by $A^{\prime} B^{\prime}$. Consequently $a$ is reduced in length and $b$ is increased in length, both by a small amount $\Delta b$, the new distances being indicated by $a^{\prime}$ and $b^{\prime}$. The image actually found on the radiogram, because of the rotation of the patient's jaw, is $P_{A}{ }^{\prime} P_{B}^{\prime}$ indicated by $\chi^{\prime}$. The difference $\Delta \chi$ between $\chi$ and $\chi^{\prime}$ is the error produced by the angle $\Delta \alpha$. Using formulas for coordinates, $\Delta \chi$ is calculated as follows (formula No. 2).*

$$
\Delta x=(a+b)\left\{\frac{\mathrm{s}+\mathrm{d} \cos \Delta \alpha}{\mathrm{T}-\mathrm{d} \sin \Delta \alpha}-\frac{\mathrm{S}}{\mathrm{T}}-\frac{\mathrm{d}}{\mathrm{a}}\right\}
$$

In this formula $S=p \cos \Delta \alpha+\mathrm{c} \sin \Delta \alpha$ and

\footnotetext{
${ }^{*} \Lambda$ copy of the derivation of $\Delta x$ can be obtained by writing to the author. $\cos =$ Cosine; $\sin =$ sine; $\tan =$ tangent.
} 


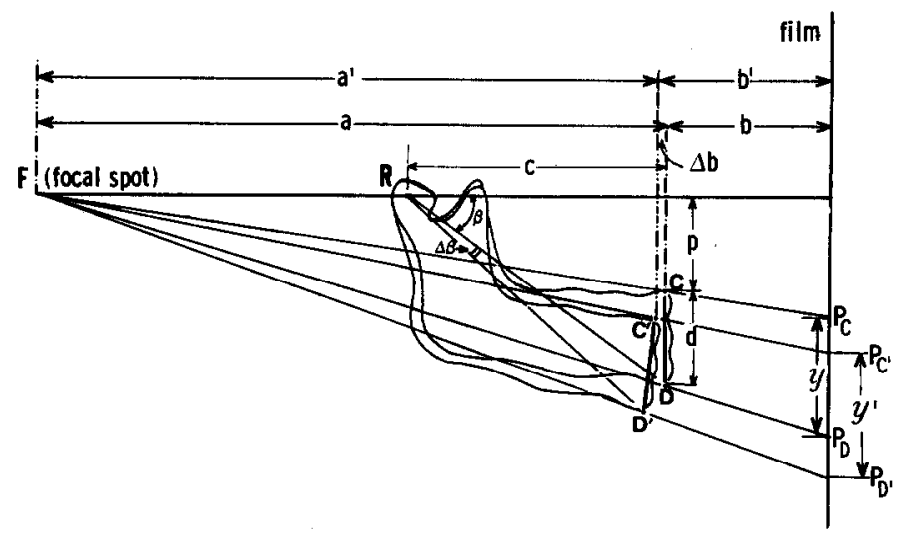

Fig. 7. Lateral view of half of mandible illustrating errors produced by differences in opening position. Image formation of part $C D$ is indicated by $y$. When patient's mandible rotates from desired position through angle $\Delta \beta$ then $C D$ becomes $C^{\prime} D^{\prime} . y^{\prime}$ is image of $C^{\prime} D^{\prime}$ on radiographic film.

Table II. Maximum geometric errors in regional measurements between serial radiograms of same subject due to deviations of angles $\alpha$ and $\beta$

\begin{tabular}{|c|c|c|c|c|c|c|c|}
\hline & & & $\mathbf{m m}^{2}$ & $\%$ & $\%$ & $\%$ & $\mathbf{m m}^{2}$ \\
\hline \multicolumn{8}{|c|}{ 30-degree oblique films } \\
\hline$a=1500$ & $x=31.50$ & $y=21.00$ & $z=661.500$ & $\Delta \chi=0.34$ & $\Delta y=0.26$ & $\Delta z=0.6$ & $\Delta z=4.0$ \\
\hline$a=3000$ & $x=30.75$ & $y=20.50$ & $z=630.375$ & $\Delta \chi=0.19$ & $\Delta y=0.12$ & $\Delta z=0.3$ & $\Delta \mathrm{z}=2.0$ \\
\hline$a=6000$ & $x=30.38$ & $y=20.25$ & $z=615.090$ & $\Delta \chi=0.13$ & $\Delta y=0.06$ & $\Delta z=0.2$ & $\Delta z=1.2$ \\
\hline \multicolumn{8}{|c|}{ 45-degree oblique films } \\
\hline$a=1500$ & $x=31.70$ & $y=21.13$ & $z=669.82$ & $\Delta x=0.30$ & $\Delta y=0.26$ & $\Delta z=0.6$ & $\Delta z=3.8$ \\
\hline$a=3000$ & $x=30.85$ & $y=20.57$ & $z=634.58$ & $\Delta \chi=0.18$ & $\Delta y=0.12$ & $\Delta z=0.3$ & $\Delta z=1.9$ \\
\hline$a=6000$ & $x=30.43$ & $y=20.28$ & $z=617.12$ & $\Delta x=0.13$ & $\Delta y=0.06$ & $\Delta z=0.2$ & $\Delta \mathrm{z}=1.2$ \\
\hline
\end{tabular}

$T=a-c+c \cos \Delta \alpha-p \sin \Delta \alpha$. Graphs were drawn with use of formula No. 2 to show relative values $\left(\frac{\Delta x}{\chi} 100\right)$ caused by horizontal deviations in the 30-degree and 45-degree projections of the patient's mandible (Figs. 4 and 5). The calculations were done for focal-spot-to-object distances of $1500 \mathrm{~mm}, 3000$ $\mathrm{mm}$, and $6000 \mathrm{~mm}$; while $\mathrm{d}$ was $30 \mathrm{~mm}$. The distance from the center of the cephalostat to the film $(b+c)$ was kept at $150 \mathrm{~mm}$. In the 30-degree oblique projection, the average of $b$ was $75 \mathrm{~mm}$ and hence $c=150-75=75 \mathrm{~mm}$. From Fig. 3 we deduced that $\mathrm{d}+\mathrm{p}=\operatorname{ctan} 30$-degrees $=43.3 \mathrm{~mm}$. In the 45-degree situation the average of $b$ was $85 \mathrm{~mm}$ and hence $c=150-85=65 \mathrm{~mm}$. If we suppose that the angle between the line from rotation center $R$ to point 0 on the film is approximately 30 degrees (Fig. 6), then it follows that in the 45-degree oblique projection $d+p=65 \tan 30$ degrees $=37.53 \mathrm{~mm}$ (Fig. 6).

From measurements made of a small sample of volunteers placed in two different cephalostats, we know that the value of $\Delta \alpha$ may range between +2.5 and -2.5 degrees. So the maximum horizontal error between two subsequent radiograms of the same subject for $\mathrm{a}=1500 \mathrm{~mm}$ is, in the 30-degree situation, $0.34 \%$ of $\chi$ (see values between vertical dashed lines in Fig. 4) and $0.30 \%$ of $\chi$ in the 45-degree situation (Fig. 5). When $\mathrm{a}=3000 \mathrm{~mm}$ and $\mathrm{a}=6000 \mathrm{~mm}$, the errors are, respectively, $0.18 \%$ and $0.13 \%$ irrespective of the angle of projection (30 and 45 degrees).

If $\Delta \mathrm{X}$ is not calculated for the mean value of $\mathrm{b}$, but instead is calculated for the maximum and the minimum values as used in Table $\mathrm{I}$, the differences are less than one thousandths of $\chi$. So there is no reason to develop graphs for different values of $b$.

\section{Vertical rotation of the mandible (ad 3)}

We know from Atwood, ${ }^{14}$ Ismail et al.,${ }^{15}$ and others that the resting face height can vary considerably. So the vertical position of the mandible of an edentulous 


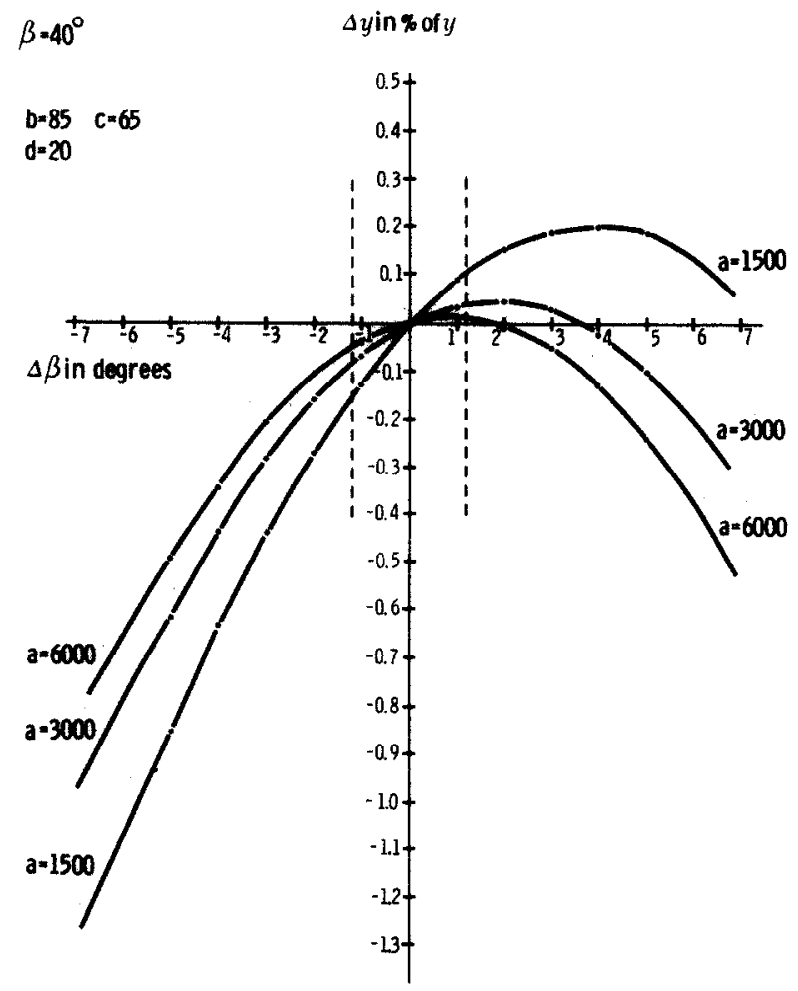

Fig. 8. Geometric error $\Delta y$ on any oblique cephalometric film expressed as percentage of $y$ due to variations in vertical position of mandible.

patient placed in a cephalostat can vary several millimeters, which results in small deviations of angle $\beta$ (Fig. 7).

When the vertical part $C D$ (Fig. 7) rotates through an angle $\Delta \beta$, the new position is represented by $C^{\prime} D^{\prime}$; $P_{C} P_{D}$ (y) is the projection of CD on the film, and $P_{C}{ }^{\prime} P_{D}{ }^{\prime}$ is that of $C^{\prime} D^{\prime}\left(y^{\prime}\right)$. The difference between $y^{\prime}$ and $y$ is indicated by $\Delta y$. There is no need to derive $\Delta y$, because the geometric situation is comparable with the horizontal situation. Therefore (formula No. 3):

$$
\Delta y=(a+b)\left\{\frac{s+d \cos \Delta \beta}{T-d \sin \Delta \beta}-\frac{S}{T}-\frac{d}{a}\right\}
$$

In this formula $S=p \cos \Delta \beta+c \sin \Delta \beta$ and $T=a-c+c \cos \Delta \beta-p \sin \Delta \beta$. With formula No. 3 the graph of Fig. 8 was drawn to show relative values $\left(\frac{\Delta y}{y} 100\right)$ as influenced by the rotation of the mandible.

Rest position measurements were made of a small sample of dentulous volunteers to calculate angle $\beta$. While the subject's porion-subnasal plane was kept in a horizontal position, angle $\beta$ ranged from 33 to 47 degrees. In Fig. 8, 40 degrees was selected for angle $\beta$.

To approximate the differences in angle $\beta$ in subsequent exposures of the mandible of the same subject, a maximum variation in the rest position of $6 \mathrm{~mm}$ seems acceptable. This results in a change in angle $\beta$, called $\Delta \beta$, of +1.2 and -1.2 degrees (see vertical dashed lines in Fig. 8). From the graph note that, with a focalspot-to-object distance of $1500 \mathrm{~mm}$, the maximum vertical error in two subsequent radiograms of the same subject is $0.26 \%$ of $y$. The figures for the other focal-spot-to-object distances are $0.13 \%$ of $y$ for $\mathrm{a}=3000 \mathrm{~mm}$ and $0.06 \%$ of $\mathrm{y}$ for $\mathrm{a}=6000 \mathrm{~mm}$. Other values of $b$ or angle $\beta$ as found in our samples influence only slightly the relative magnitude of $\Delta y$.

\section{Errors in area measurements of the edentulous mandible}

Table II shows the influence of the maximum error that is caused by deviations in the position of the mandible on a rectangular area that measures $30 \mathrm{~mm}$ and $20 \mathrm{~mm}$ in the horizontal and vertical directions, respectively. From the graphs of Figs. 4 and 5, we took as the values for $\Delta \chi$ the maximum difference that could occur in the range of $\Delta \alpha$ between -2.5 and +2.5 degrees (Fig. 7).

The values of $\Delta y$ were read from Fig. 8 as the maximum difference in the range of $\Delta \beta$ between -1.2 and +1.2 degrees.

The magnitude of the relative error for the area is:

$$
\frac{\Delta z}{z}=\frac{\Delta x}{x}+\frac{\Delta y}{y}
$$

The significance of the values $\frac{\Delta z}{z}$ for a clinical investigation can only be determined when the effects of the other variables in the technique are known.

\section{CONCLUSIONS}

The object of this study was to analyze the geometric errors that occur in oblique cephalometric projections of the edentulous mandible. From the graphs it will be clear that the choice of the distance between focal spot and object depends on the magnitude of the error that one is willing to accept.

Comparing measurements on oblique radiograms of one subject. The enlargement factor (formula No. 1) can be kept practically constant when the difference in the opening position of the mandible in relation to the porion-subnasal plane does not exceed $6 \mathrm{~mm}$ at the moment of exposure. When exposures of a subject's 
mandible within the range of the rest position are made, the calculated values of $\Delta \chi$ and $\Delta y$ amount to a few thousandths of $\chi$ and $y$.

To ensure the accuracy of this radiographic measuring method it is important to mimimize the horizontal and the vertical deviations. Therefore the following items are important.

1. The brackets with the ear fixation pins of the cephalostat show no tolerance.

2. When in use, the pins should be pressed as close into the meatus as possible without causing pain.

3. The inter-earpin distance should be kept constant in subsequent radiograms of the same patient.

4. The position of the mandible is at resting vertical dimensions.

Comparing measurements on oblique radiograms of different subjects. The enlargement factor may vary because of the following.

1. Anatomic variations caused by the shape of the mandible

2. Variation in the vertical position of the mandible at rest caused by differences in the anatomic shape of the skull as distinct from individual variations in the rest position

ad 1 . These variations result in different values for $b$. In Table I the influence of $b$ on the enlargement is shown.

ad 2. These variations result in different values for angle $\beta$. In the sample of volunteers, $\beta$ ranged from 33 to 47 degrees.

Assuming that the radiographed section is always parallel to the film, these variations result in an error of $1 \%, 0.5 \%$, and $0.2 \%$ of $y$ with a focal-spot-to-object distance of $1500 \mathrm{~mm}, 3000 \mathrm{~mm}$, and $6000 \mathrm{~mm}$, respectively. There is a linear interrelationship between these errors and the deviations from the mean value of $\beta$ (40 degrees).

To minimize the variation in the enlargement factor between subjects it is important to standardize the vertical inclination of the lower border of the mandible. To avoid overprojection of the base of the skull, an inclination of approximately 15 degrees between the lower border of the mandible and the horizontal porion-subnasal plane was found to give acceptable results. In order to position the lower border of the mandible at this inclination, a special support with fixation unit was developed. ${ }^{16}$

In part II the clinical application of this oblique cephalometric technique will be studied.

\section{SUMMARY}

Geometric errors that occur in oblique cephalometric radiographic projections of the edentulous mandible were calculated for different focal-spot-to-object distances $(1500,3000$, and $6000 \mathrm{~mm})$. The horizontal errors from tolerance of the porion and nasion fixation in the cephalostat were calculated. The same was done for the vertical errors caused by the variability of the rest position. From the results of this study it can be concluded that the distance between focal spot and object should be as large as possible. It was also found that it is important to minimize the differences in enlargement that may occur on radiograms of different subjects because of changes in the position of the mandible. This position can be standardized by the use of an individual support. ${ }^{16}$

This material was prepared in cooperation with the Department of Oral Radiology.

\section{REFERENCES}

1. Wical, K. E., and Swoope, C. C.: Studies of residual ridge resorption. Part I. Use of panoramic radiographs for evaluation and classification of mandibular resorption. J PROSTHET DENT 32:7, 1974.

2. van Waas, M. A. J.: Een röntgenologisch onderzoek naar de resultaten van autologe bottransplantaten. Netherlands Dental Journal 86:25, 1979.

3. van $\Lambda$ ken, J.: Geometrical errors in lateral skull $\mathrm{x}$-ray projections. Ned Tijdschr voor Tandheelk 70:1, 1963.

4. van Aken, J.: Panoramic x-ray equipment. J Am Dent Assoc 86:1050, 1973.

5. Lund, T. M., and Manson-Hing, L. R.: A study of the focal troughs of three panoramic dental $\mathrm{x}$-ray machines. Part II. Image dimensions. Oral Surg 36:647, 1975.

6. Tallgren, A.: Changes in adult face height, due to ageing, wear and loss of teeth and prosthetic treatment. Acta Odontol Scand 15(Suppl 24):1, 1957.

7. Tallgren, A.: The continuing reduction of the residual alveolar ridges in complete denture wearers: A mixed-longitudinal study covering 25 years. J Prosthet Dent 27:120, 1972.

8. Tallgren, A., Lang, B. R., Walker, G. F., and Ash, Jr., M. M.: Roentgen cephalometric analysis of ridge resorption and changes in jaw and occlusal relationship in immediate complete denture wearers. J Oral Rehabil 7:77, 1980.

9. Carlsson, G. E., and Persson, G.: Morphologic changes of the mandible after extraction and wearing of dentures. Odont Revy 18:27, 1967.

10. Atwood, D. A., and Coy, W. A.: Clinical, cephalometric, and densitometric study of reduction of residual ridges. J Prosthet DeNI 26:280, 1971.

11. Cartwright, L. J., and Harvold, E.: Improved radiographic results in cephalometry through the use of high kilovoltage. Can Dent Assoc J 20:261, 1954.

12. Barber, Th. K., Pruzansky, S., and Kindelsperger, R.: An evaluation of the oblique cephalometric film. J Dent Child, 28:94, 1961. 
13. Winter, C. M., Woelfel, J. B., and Igarashi, T.: Five-year changes in the edentulous mandible as determined on oblique cephalometric radiographs. J Dent Res 53:1455, 1974.

14. Niwood, D. A.: A cephalometric study of the clinical resorption of the mandible. Part I. The variability of the clinical rest position following the removal of occlusal contacts. J Prosi HEI Dent 6:504, 1956

15. Ismail, Y. H., George, W. A., Sassouni, V. and Scott, R. H.: Cephalometric study of the changes occurring in the face height following prosthetic treatment. Part 1. Gradual reduction of both occlusal and resi face height. J Prosthet Dent 19:321, 1968.
16. Steen, W. H. A.: A positioning device for the edentulous mandible in extraoral radiography. J Prosthry Dent 48:257, 1982.

Reprint requests to:

Dr. W. H. A. STEEN

SCHOOL OF DENTISTRY

UNIVERSITY OF UTRECHT

SORBONNELANN 16

3508 TB UTRECHT

THE NETHERLANDS

\section{AADR PROSTHODONTIC ABSTRACT}

\section{Single crystal sapphire endosteal implants: Two-year clinical results}

\section{L. Koth, R. V. McKinney, and Q. B. Davis}

Medical College of Georgia, Augusta, Ga.

Endosseous dental implants have been evaluated clinically with conflicting results regarding the interrelation among implant design, composition, and tissue compatibility. This is due in a large part to a lack of uniform and practical parameters for evaluating an implant's success. The purpose of this study was twofold: (1) to establish a longitudinal study for the clinical evaluation of a single crystal screw-type sapphire endosteal implant, and (2) to establish clinical parameters that could be universally applied to evaluate an implant's success. This article reports the two-year

Supported by Johnson \& Johnson Co., Grant No. 10-12-02-1260-63, and Kyocera International, Inc.

Reprinted from the Journal of Dental Research (62 [Special Issue], 1983 ((Abstr No. 1111) with permission of the author and the editor. results of the study. A total of 24 implants were placed in the mandibles of 15 patients by two oral surgeons. After 6 weeks healing, fixed prostheses with implants serving as distal abutments were placed. The implants were evaluated every 3 months for the first year and then yearly using the following parameters: bleeding index, plaque index, quantitation of crevicular fluid volume, mobility, radiographic interpretation, and patient comfort. Criteria for success were: bleeding index ( 2 or less), crevicular fluid volume (10 or less), mobility ( 2 or less), radiographic index ( 2 or more positive ratings), and absence of pain; any implant failing in three or more criteria were judged as failures. The two-year results yielded a $95 \%$ success rate for the implants that served as abutments and a $75 \%$ success rate for all implants. After 2 years, the parameters for evaluation seem to be indicative of implant behavior. 